TITLE:

\title{
Fabrication of gated niobium nitride field emitter array
}

AUTHOR(S):

Gotoh, Y; Kashiwagi, Y; Nagao, M; Kondo, T; Tsuji, H; Ishikawa, J

\section{CITATION:}

Gotoh, Y ...[et al]. Fabrication of gated niobium nitride field emitter array. JOURNAL OF VACUUM SCIENCE \& TECHNOLOGY B 2001, 19(4): 1373-1376

ISSUE DATE:

2001-07

URL:

http://hdl.handle.net/2433/39779

RIGHT:

Copyright 2001 American Vacuum Society 


\title{
Fabrication of gated niobium nitride field emitter array
}

\author{
Y. Gotoh, ${ }^{\text {a) }}$ Y. Kashiwagi, M. Nagao, ${ }^{\text {b) }}$ T. Kondo, H. Tsuji, and J. Ishikawa \\ Department of Electronic Science and Engineering, Kyoto University, Yoshida-honmachi, Sakyo-ku, \\ Kyoto 606-8501, Japan
}

(Received 20 September 2000; accepted 21 May 2001)

\begin{abstract}
We have fabricated a gated niobium nitride field emitter by the transfer mold method. A silicon mold was fabricated by anisotropical etching, followed by oxidation of the mold and deposition of niobium nitride. Niobium nitride thin films were prepared by ion-beam-assisted deposition. After attaching the sample to a glass substrate, the silicon mold was removed by mechanical and wet etching. A molybdenum thin film was then deposited by electron-beam evaporation. Formation of a gate aperture and an insulating silicon dioxide layer were performed by wet etching of only the apex, with other regions being protected by a photoresist layer. An electron emission test of the fabricated emitter array was performed in a high vacuum. We confirmed electron emission of up to $0.1 \mu \mathrm{A}$ at the emitter-gate voltage of $30 \mathrm{~V}$. (C) 2001 American Vacuum Society.
\end{abstract}

[DOI: $10.1116 / 1.1385913]$

\section{INTRODUCTION}

Transition-metal nitrides and carbides possess high melting points, chemically inert surfaces, high electrical conductivities, and relatively low work functions. ${ }^{1,2}$ These properties meet the requirements of the cathode material, which is exposed to the severe conditions of Joule heating and ion bombardment. Generally, carbides ${ }^{3}$ attract much interest because of their low work functions, and nitrides have attracted less interest, except for titanium nitride. Transition-metal nitrides have higher heats of formation than the carbides, ${ }^{4}$ and higher stability of the material is therefore expected with the nitrides. We have been investigating the film property and the electron emission properties of transition-metal nitrides. ${ }^{5-7}$ We found that niobium nitride $(\mathrm{NbN})$ with the stoichiometric nitrogen composition exhibits a stable electron emission, ${ }^{7}$ which agrees with the results of the evaluation of bulk nitride emitters. ${ }^{8}$ We also confirmed a greater resistance against ion bombardment of this material. ${ }^{6}$ The emission test was performed, however, with a simple diode structure that consists of nitride-deposited silicon cones and a counter electrode. To demonstrate the feasibility of the nitride as a cathode material, establishing a fabrication process of nitride emitters is necessary. In the present article, we fabricated a gated $\mathrm{NbN}$ field emitter array by the transfer mold method. ${ }^{9}$ This method has such an advantage that this process has no preference of cathode material. In depositing the nitride, we adopted ion-beam-assisted deposition by which low-temperature growth of the compound material is expected.

\section{FABRICATION OF GATED NBN EMITTER}

\section{A. Flow of fabrication process}

The fabrication process is divided into two major processes: the formation of a $\mathrm{NbN}$ emitter and the formation of

\footnotetext{
a) Author to whom all correspondence should be addressed; electronic mail: ygotoh@kuee.kyoto-u.ac.jp

${ }^{b}$ Present address: National Institute of Advanced Industrial Science and Technology, Tsukuba 305-8568, Japan.
}

a gate electrode. Figures $1(\mathrm{a})-1(\mathrm{~h})$ show a schematic drawing of the device undergoing processing. Figures 1(a)-1(e) are the emitter formation process, and Figs. 1(f) and 1(g) are the gate formation process.

\section{B. Formation of $\mathrm{NbN}$ emitter by transfer mold method}

First, the thermal oxide $\left(\mathrm{SiO}_{2}\right)$ layer with a thickness of 1 $\mu \mathrm{m}$ on the $p$ - $\mathrm{Si}(100)$ substrate was patterned. A positive tone photoresist, OFPR800 (Tokyo Ohka, 20cp), was spin coated. A mask pattern consists of 26 circles, each with a diameter of $3 \mu \mathrm{m}$. The 25 circles were arranged in a square having sides made by five circles of $20 \mu \mathrm{m}$ intervals. Another circle was located beside the square to show the orientation of the sample. After developing the photoresist, the $\mathrm{SiO}_{2}$ layer was etched off by buffered hydrofluoric acid (BHF), resulting in the formation of 26 holes [Fig. 1(a)]. In Fig. 1, the process for one circle is illustrated.

The silicon substrate under the $\mathrm{SiO}_{2}$ mask was anisotropically etched by tetramethylamoniumhydroxide (TMAH, Tokyo Ohka NMD-3). Due to the low etching rate for the $\mathrm{Si}(111)$ plane, pyramidal pits were formed [Fig. 1(b)]. The $\mathrm{SiO}_{2}$ layer at the surface was removed, and then the entire surface was again oxidized in a furnace with oxygen flow [Fig. 1(c)]. Oxidization was performed in a dry atmosphere for $3 \mathrm{~h}$, then in a wet atmosphere for $7 \mathrm{~h}$. The resulting film thickness was about $600 \mathrm{~nm}$. This oxidized layer acts as an insulator between the emitter and the gate.

The mold was filled with $\mathrm{NbN}$ film by ion-beam-assisted deposition [Fig. 1(d)]. ${ }^{6}$ The deposition conditions are listed in Table I. There are some difficulties in filling material in the pit, because the metal vapor and the ion beam are incident on the substrate from different directions. We set the substrate to have a similar angle (nominally $22.5^{\circ}$ ) to the evaporator and the ion source, to avoid only one component of the material sticking to the face of the mold, as shown in Fig. 2. Monitoring of the deposited film was performed by analyzing the film that was simultaneously deposited on a flat substrate. The nitrogen composition in the film was 


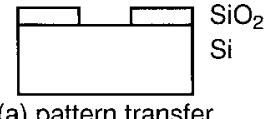

(a) pattern transfer

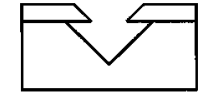

(b) mold formation

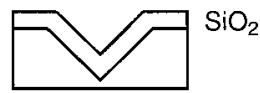

(c) formation of insulator

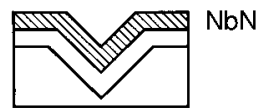

(d) NbN deposition

Emitter formation

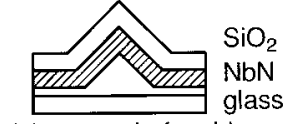

(e) removal of mold

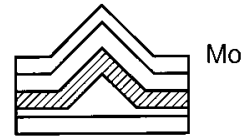

(f) gate deposition

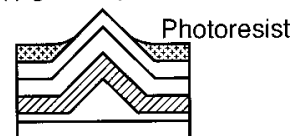

(g) spin coat of photoresist

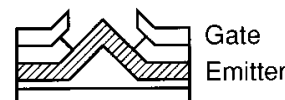

(h) gate aperture formation

\section{Gate formation}

FIG. 1. Fabrication process of a gated niobium nitride emitter is shown.

evaluated by Rutherford backscattering spectrometry. We adjusted the deposition conditions so that the film on the flat substrate had a stoichiometry nitrogen composition. Although it is difficult to estimate the composition of the film inside the mold on each side of the pyramidal mold, sufficient nitrogen was considered to be incorporated in the film on each side. This was suggested by the adequate resistance of the emitter against the BHF exposure as described later. After depositing a $300 \mathrm{~nm} \mathrm{NbN}$ film, $\mathrm{Nb}$ evaporation without ion bombardment was performed to ensure the stiffness of the device after the removal of the silicon mold. The nominal total thickness was about $1 \mu \mathrm{m}$. Figure 3(a) shows a scanning electron micrograph (SEM) image of the cross-sectional view of the $\mathrm{NbN}$-deposited mold. The outermost layer is $\mathrm{NbN}$, and the internal layer is $\mathrm{SiO}_{2}$. The $\mathrm{NbN}$ film filled the mold, but the thicknesses of the $\mathrm{NbN}$ films differed between the faces in the mold, as expected. As shown in Fig. 2, metal vapor comes from just below, whereas the nitrogen ion beam comes from the right-hand side. This means that the flux of the metal vapor becomes higher for the right-hand side face of the mold, and that the flux of the nitrogen ions becomes higher for the left-hand side face of the mold. Thus, the layer formed on the left-hand side face was fabricated at a lower deposition rate with a higher ion-current density, resulting in the formation of a thinner layer on the left-hand side face as compared with the right-hand side face.

TABLE I. Deposition conditions of NbN emitter layer by ion-beam-assisted deposition are shown.

\begin{tabular}{cc}
\hline \hline Term & Condition \\
\hline Nitrogen ion energy & $600 \mathrm{eV}$ \\
Arrival rate ratio $(\mathrm{N} / \mathrm{Nb})$ & 2.0 \\
Substrate temperature & $500^{\circ} \mathrm{C}$ \\
Nominal thickness & $300 \mathrm{~nm}$ \\
\hline \hline
\end{tabular}

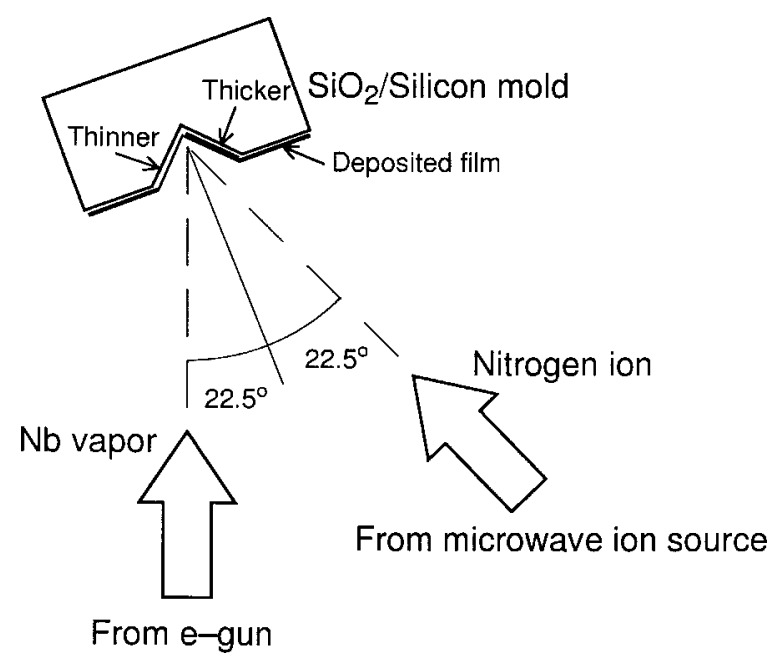

FIG. 2. Angle of the sample in the ion-beam-assisted deposition system and resulting film thickness on the different side of the mold are shown. The difference in thickness is exaggerated.

The sample was attached to a glass plate with a vacuum sealant (Varian, Torr Seal) as an adhesive. After the attachment, the silicon mold was removed, first by mechanical etching and then by wet etching [Fig. 1(e)]. The initial stage of the wet etching was performed with potassium hydroxide $(\mathrm{KOH})$. This etchant was used because tetramethyl ammonium hydroxide (TMAH) did not show start of etching. The etchant was changed to TMAH after the etching started, because $\mathrm{KOH}$ dissolves the $\mathrm{SiO}_{2}$ layer.

\section{Formation of gate aperture}

A molybdenum (Mo) thin film with a thickness of $300 \mathrm{~nm}$ was electron beam evaporated at room temperature as a gate electrode [Fig. 1(f)]. Figure 3(b) shows the SEM image of the Mo covered sample. After deposition of Mo, the sample was spin coated with a photoresist (OFPR800, 50cp) [Fig. $1(\mathrm{~g})]$. The revolution and coating time were adjusted to 3000 $\mathrm{rpm}$ and $40 \mathrm{~s}$, respectively, so that the photoresist layer covers the entire sample except the apex of the pyramids. The sample was dipped into phosphoric nitric acid without developing the photoresist, to form a gate aperture [Fig. 1(h)]. Subsequent etching of the oxide layer was performed with BHF. During the etching process of the $\mathrm{SiO}_{2}$ layer, the emitter layer was exposed to the BHF. The preliminary test showed that $\mathrm{NbN}$ was not attacked by BHF, although elemental $\mathrm{Nb}$ was seriously attacked. Figure 3(c) shows the SEM image of the completed sample. Some of the gate electrodes were lost due to peeling off, but most of the gate aperture formed successfully. Figure $3(d)$ is a magnified view for one emitter. A gate aperture of about $1 \mu \mathrm{m}$ was formed.

\section{ELECTRON EMISSION TEST}

\section{A. Measurement system}

The measurement chamber and circuit used in the present study is that used for evaluating Spindt-type cathodes in our laboratory. ${ }^{10}$ The gate electrode was grounded, and the emitter was negatively biased. An external collector, that is a 


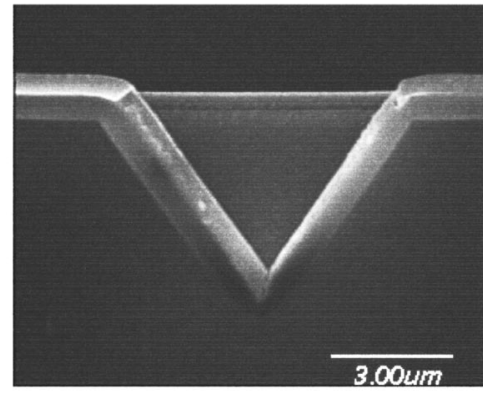

(a)

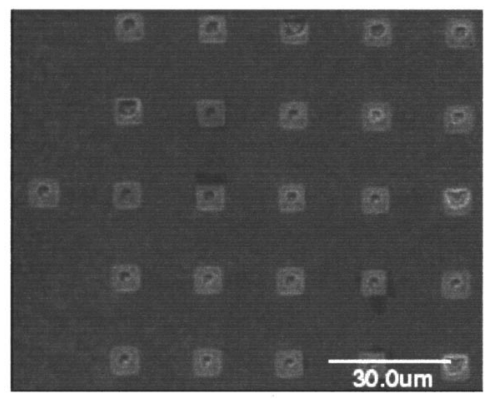

(c)

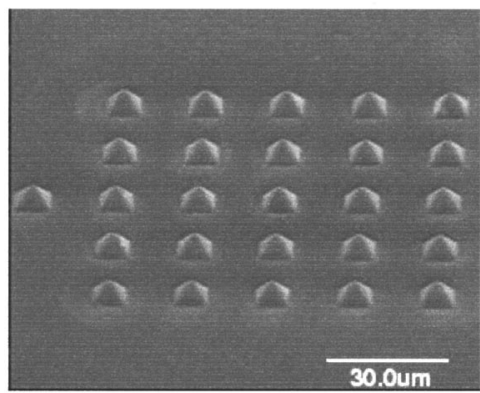

(b)

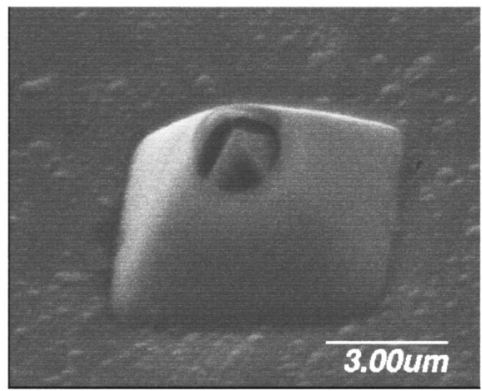

(d)
FIG. 3. SEM of the fabricated device is shown. (a) $\mathrm{NbN}$-deposited mold, (b) Mo-deposited emitter array, (c) after gate-aperture formation, and (d) close-up of the fabricated emitter are presented. phosphor-coated conductive glass plate, was positively biased to $200 \mathrm{~V}$. The gap between the sample and the collector was about $5 \mathrm{~mm}$. In the present setup, the electric field between the gate and the collector was sufficiently weak so as not to cause electron emission from the gate electrode. The chamber was evacuated by a turbomolecular pump to $10^{-5}$ $\mathrm{Pa}$. The collector current and gate current were monitored. Through a view port, we observed the emission pattern.

\section{B. Electron emission characteristics}

Figures 4(a) and 4(b) show a typical example of the current-voltage characteristics and the Fowler-Nordheim plot of the fabricated emitter, respectively. The currentvoltage characteristics were first those represented by the rhombus symbols, but after some occasional arcs, changed to those represented by the circle symbols. The emission threshold shifted from 17 to $22 \mathrm{~V}$. However, between these arcs, the current was fairly stable. The collector current reached $0.1 \mu \mathrm{A}$ at the gate-emitter voltage of $30 \mathrm{~V}$. The degradation would probably be due to the destruction of microprotrusions. The gate current was as large as $100 \mu \mathrm{A}$, which includes the emitted current to the gate and the leakage current.

The luminescence was observed just above the device, which suggests the emission was from the fabricated device. Together with this spot, some additional spots were occasionally observed around the major spot. The major spot was stable and did not change its position. The emission pattern showed that the emission was limited by the presence of the gate electrode. This result agrees with the large current observed for the gate electrode.
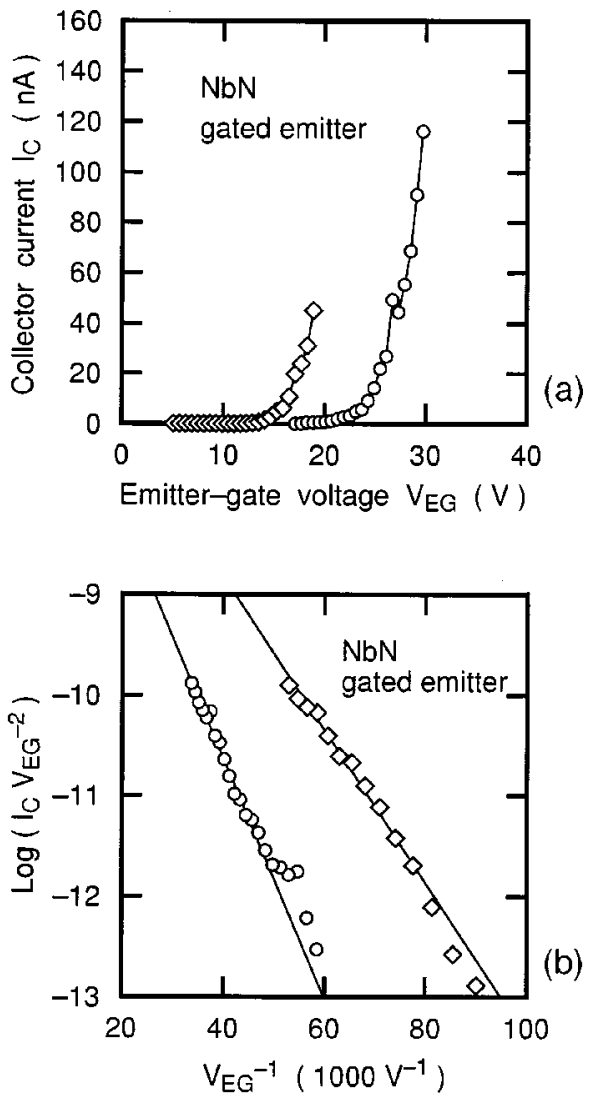

FIG. 4. Electron emission characteristics of the fabricated gated $\mathrm{NbN}$ emitter are shown. (a) Current-voltage characteristics and (b) Fowler-Nordheim plot are presented. 


\section{DISCUSSION AND SUMMARY}

We have successfully fabricated a $\mathrm{NbN}$ field emitter array by the transfer mold method. Electron emission from the device was confirmed by the current and luminescence of the phosphor-coated collector. In the present device-anode geometry, we could not resolve the individual emission sites, thus we could not tell how many microtips were active. The number of the active microtips was considered to be a dozen or less, because some emitters did not have the gate which could apply the extraction voltage effectively. We consider that the emission occurred not only at the apex, but also, at the ridgeline. The postoxidation in the mold formation process makes the ridgeline of the mold sharper, resulting in the sharpened ridgeline of the $\mathrm{NbN}$ emitter. This is attributable to the large gate current. Emission from the gate electrode is not plausible, because the electric field which was applied to the gate was, as described earlier, too weak to yield electron emission.

We still have the following difficulties. First, the $\mathrm{NbN}$ layer was nonuniform due to the fixed directions of ion and metal vapor incidence. This could be solved by introducing the substrate rotation system. Second, the NbN film contained stress and was occasionally warped, splitting the apex of the emitter. Optimizating the deposition parameters and introducing substrate rotation is necessary. Third, the gate electrode peels off during the process of forming the gate aperture. Formation of the stiff gate electrode, for example, by sputtering, is necessary. In spite of all the difficulties previously described, a gated $\mathrm{NbN}$ field emitter array was fabricated. Further optimization of the formation process may improve the emission property.

\section{ACKNOWLEDGMENTS}

This work was partly supported by the Ministry of Education, Culture, Sports, Science, and Technology, through a
Grant-in-Aid for Encouragement of Young Scientists, No. 09750016, and a Grant-in-Aid for Scientific Research B, No. 11555006. The composition of the nitride films were evaluated by Rutherford backscattering spectrometry with the "Experimental System for Ion-Beam Analysis," Quantum Science and Engineering Center, Kyoto University. The authors would like to thank the staff of the QSEC for encouragement during the course of the work. They also thank Professor Susumu Noda, Department of Electronic Science and Engineering, Kyoto University, for the assistance in microfabrication. The SEM images were taken with a fieldemission SEM which is equipped at the Kyoto University Venture Business Laboratory.

${ }^{1}$ L. E. Toth, Transition Metal Carbides and Nitrides (Academic, New York, 1971).

${ }^{2}$ V. S. Fomenko, Handbook of Electron Emission Property (Nisso Tsushinsha, Wakayama, 1970) (in Japanese).

${ }^{3}$ W. A. Mackie, T. Xie, and P. R. Davis, J. Vac. Sci. Technol. B 17, 613 (1999).

${ }^{4}$ O. Kubaschewski and C. B. Alcock, Metallurgical Thermochemistry, 5th ed. (Pergamon, Oxford, 1979).

${ }^{5}$ M. Nagao, Y. Fujimori, Y. Gotoh, H. Tsuji, and J. Ishikawa, J. Vac. Sci. Technol. B 16, 829 (1998).

${ }^{6}$ Y. Gotoh, M. Nagao, T. Ura, H. Tsuji, and J. Ishikawa, Nucl. Instrum. Methods Phys. Res. B 148, 925 (1999).

${ }^{7}$ M. Nagao, Y. Gotoh, T. Ura, H. Tsuji, and J. Ishikawa, J. Vac. Sci. Technol. B 17, 623 (1999).

${ }^{8}$ M. Endo, H. Nakane, and H. Adachi, J. Vac. Sci. Technol. B 14, 2114 (1996).

${ }^{9}$ M. Nakamoto, K. Ichimura, T. Ono, and Y. Nakamura, Eighth International Vacuum Microelectronics Conference, IVMC'95, Portland, Oregon, 31 July-3 August 1995, p. 186.

${ }^{10}$ Y. Gotoh, K. Utsumi, M. Nagao, H. Tsuji, J. Ishikawa, T. Naktani, T. Sakashita, and K. Betsui, J. Vac. Sci. Technol. B 17, 604 (1999). 